\title{
INTERVIEW
}

\section{Words in culture: An interview with Anna Wierzbicka*}

Andor: Thank you very much for accepting my call for this interview. I am honored to have the chance to record it in Landau, Germany, just before the LAUD symposium, which is topically devoted to intercultural pragmatics, a field which is closely related to the type of research you have been doing during the past few decades: research in a universalist approach to lexical semantics and (may I also say) lexical pragmatics.

I would like to start this interview with a general question. People like Cliff Goddard, who has been one of your closest co-workers for a number of years now, have expressed a view (Goddard 2003), that your lexicalist approach to studying language shows considerable indebtedness to the legacy of Whorf and Sapir, although he also pointed to the differences. In the study I am referring to, Goddard expresses a view that even cognitivists like Langacker show affinity with Whorf concerning the origins of their theoretical approach to understanding the basic functions and operation of language. Now, in an interview I had with Ronald Langacker in 2005, he expressed his sympathy with your cognitive-lexicalist approach (cognitive lexicographic program, as he put it, and your natural semantic metalanguage program) (Andor 2005a, 363). He certainly did this with reason. Do you also sympathize with cognitive grammar as outlined and researched by Langacker and co-workers? Would you, yourself, consider your research program to be neo-Whorfian in its nature and scope?

* This interview was recorded in Landau, Germany, on 26 March, 2006, a day before the 31st International LAUD Symposium on Intercultural Pragmatics organized by the University of Koblenz, Landau (27-30 March, 2006). In preparing the transcript I intended to keep the many discourse markers used by Anna Wierzbicka and myself during our conversation, with the aim to retain the original tone of the interview. 
Wierzbicka: Thank you very much. First of all, thank you for inviting me and giving me this chance to express my views on a number of topics. You raised, of course, already quite a number of questions, on Whorf and Sapir, for instance. As we all know, Whorf's and Sapir's theories have been interpreted by many different scholars in many different ways. So, in a sense, it's dangerous to say, I agree or I don't agree with Whorf, because we have to explain what exactly it is that you agree with or you don't agree with. But yes, certainly, in my experience, as someone who has been trying to understand various aspects of meaning embedded in the languages, well, I think, I see that languages differ enormously in the interpretation of the world, and therefore, that they carry with them a certain world view, and you might say, yes, this is Whorf and Sapir. You could also say this is Humboldt, and it goes further back. In fact, you find similar ideas further back, too, in Leibniz, to some extent, and so on. At the same time, some of Whorf's formulations are very extreme, and when he talks about the impressions we have of the world he uses the word kaleidoscope as if there were no categories which are given the same in our languages. Well, we are interested in how languages differ but also in what they have in common; we are interested in language universals. And we find that to understand how language works and how it's related to thought, we really have to bear those two aspects in mind at the same time and ask what does not differ from language to language, and what does differ. So if we turn to this image of the kaleidoscope, the kaleidoscopic flow of different images, and so on, well, we find certain categories which do not depend on language. Like, for example, I did basic categorization distinguishing who from what. There is a distinction drawn between who and what. This is not something which depends on the particular language or culture. Well, presumably, this distinction is somehow innate. It's given in all languages. At the same time, we find huge differences in all areas, for example in the way emotions are categorized in different languages and the way values are categorized, or further on, mental predicates, and so on. And furthermore, something which for us has been very important is the idea that if we want to compare languages at all, if we want to identify some differences between them, well, for this we need certain tools which are language independent. If we did not have universals of language and thought, I think we would not be able to talk about the differences either. We would have no tools. And actually, there is a nice quote somewhere in Whorf's writings, which is very seldom, if ever, recalled. He, too, talks about 'foundational notions' - I think he used this word - which are the same in all languages and which underlie all the different ideas, configurations of elementary units. Well, he does not actually use the word configurations, but I think he does use 'building blocks' or 'foundational concepts', or something like this.

Andor: My second question also concerns a general issue. Your approach to the linguistic description of the lexis (or lexicon) - in its core - is basically semantic. The model that is now associated with your name-Natural Semantic Metalanguage, NSM - carries this scope in its name. Still, the way you have developed your theory since the early times, the time when Lexicography and Conceptual Analysis was published (Wierzbicka 1985), has revealed a clear devotion to lexical pragmatics. Especially in the way you discussed conceptualization and categorization, the role of prototypicality in lexical representation, the critical role of various types of knowledge (and knowledge acquisition), such as encyclopaedic vs. lexical, and their differences from technical and other types of knowledge, etc., the interrelatedness of all these domains in lexical representation, storage, and even retrieval. I think we can agree to say that these issues carry 
at least as much pragmatic weight as they are semantically based. Where would you draw - if at all you think that it would be necessary - the borderlines between lexical semantics and lexical pragmatics?

Wierzbicka: Well, I'm not sure how to draw the lines, because these words, 'semantics' and 'pragmatics' still are, in a sense, artificial technical terms. We can draw the lines wherever we want to. There is nothing given, you know, essential about the term 'pragmatics', and if somebody wants to use the word 'pragmatics' in a different way than I do, I don't mind. The important thing from my point of view is how to describe all the knowledge that the native speaker of a language has to have about a particular word to be able to use this word in the way that other native speakers of this language use it. And as we work on individual words, we often discover that there is a tremendous amount of information encoded in a word. Some people don't like the word 'encoded'. Again, I don't necessarily have to rely on the word 'encoded'. But there is a tremendous amount of knowledge that native speakers have and the question is what is the best and most effective way of presenting this knowledge. As I see it, many people who draw fairly, sort of strict, sharp lines between what some people call semantics on the one hand and pragmatics on the other hand, tend to exclude a large part of knowledge which is necessary for behaving in the work as a native speaker, because this, they say, does not matter, because this is pragmatic. But to me this is not good enough.

Andor: This is exactly what I want to hear. This is exactly why I asked you this question, because I knew that you would be approaching it from this point of view, which I highly appreciate.

Wierzbicka: Well, I'm very glad to hear this. It seems to me that probably at this point it would be useful to talk about some examples. I didn't know that you were going to ask me this question, because I would have prepared a few examples, and I don't have them at the moment. Because we find that the knowledge encoded in words is often extremely rich and complex, and indeed, there may be aspects of this knowledge, sort of aspects of different kinds, and to find the optimum representation of the whole thing, well, quite possibly some aspects need to be treated in some ways and some other aspects in another way. So it's not a set of features, sort of listed with always the same set. First of all, it's not a set of features at all. Because, as you know, the way we see it, a semantic representation, a representation of the cultural knowledge linked with a particular word to be useful, should be stated in a way which people can understand. So I don't think there should be any sort of technical a priori assumptions there. You have to find out, in a sense, by trial and error, what the most effective, the most optimal way of representing these aspects of knowledge is. But I wouldn't exclude anything. If there are certain aspects of knowledge which native speakers, as native speakers, have in connection with a particular word, well, this needs to be simply included in the description of this word, and also in dictionaries. Of course, there are sort of pragmatic and practical considerations that in a dictionary to be used by so-called ordinary people for the purposes of ordinary communication, well, sometimes you have to abbreviate, you have to choose, and so on. But ideally, in fact, even to abbreviate and to choose in an optimal way, in relation to particular practical goals, it is good to have a complete picture of the knowledge that native speakers have, that they associate with a particular word.

Andor: My next question concerns the practical issue of the nature of the dictionary representation of the 'amalgam' of features (and knowledge about) words and expres- 
sions. To what extent should dictionary definitions be semantic and pragmatic? To what extent should modern dictionaries be frame based, along the lines of research done by, for instance, Fillmore's FrameNet Project? Personally, I would agree that in our electronic age cognitive-based contextual knowledge should somehow be represented in dictionaries much more extensively than it was earlier, and cross referencing on the basis of empirically tested, relevant lexical constituents of frames (and also of scripts) should be guaranteed. But what's the best way of carrying this out? How to decide, for instance, on the required depth of the representation of contextual information? I believe your work could be usefully applied here. At the time of reading your classical monograph, Lexicography and Conceptual Analysis in 1985, and other pieces of your work published already around those years and later, my judgment was that you supported the idea that dictionaries be more frame based, that they should represent encyclopaedic knowledge in radically greater detail than earlier. However, in later years, with the NSM framework gaining more and more ground, I seem to experience a change in this view. In 1996, in Semantics: Primes and Universals, you expressed that dictionary definitions should not be overburdened with an extensive representation of encyclopaedic knowledge, as this kind of knowledge is not representative of the body of general knowledge of native speakers. Instead, dictionary definitions should reflect, represent the folk concepts encoded in the given language by its native speakers (Wierzbicka 1996, 263), using very simple language, perhaps the language of the NSM lexis. What is your current view on this issue? What would you advise to editors of dictionaries? Are you satisfied with the kind of modern, corpus-based dictionaries at our disposal today, which are even published in CD-ROM versions, frequently with a corpus sample available for testing the correctness and relevance of their definitions?

Wierzbicka: Again, this is a set of complex questions. In principle, I think, our attitude remains the same about the so-called encyclopaedic knowledge. Well, it should be represented in the dictionary, but the question is what we mean by encyclopaedic knowledge. Indeed, I think there was always a position that what we are after is the knowledge of all ordinary native speakers. So it is cultural knowledge, folk knowledge, rather than technical knowledge and the knowledge of scientists. So on this point I see that nothing has changed. But, you know, there is certainly a change linked with the use of corpora. I think that the use of corpora has really revolutionalized linguistics; it has also revolutionalized lexical semantics. And in our recent work we use corpora extensively. For example, maybe I can just draw here on my more recent work. I did a general study on the English word evidence (Wierzbicka in press), which I regard as one of the sort of particularly important English words, because it's used constantly, it's one what I call 'English cultural keywords'. So we try to say what the word evidence means. Now, to understand this question about the meaning of evidence, I think, I would look certainly at a large number of examples. I will pull out literally hundreds of examples of sentences using the word evidence from the corpora. And, first of all, corpora give us a huge database. But they also allow us to deal with a crucial problem of lexical semantics, which is polysemy. We have to decide how many different meanings a word has, because without this we cannot test our definitions in context. And of course, our definitions have to be, in a sense, frame-based, because since we are using examples of sentences, we need a kind of analysis of paraphrase of a word like evidence, which would fit in sentences in context. In the case of a word like evidence, and probably most other words, you can't find one paraphrase which would fit all the contexts. So you are forced to recognize polysemy. Of course, you try to recognize 
the minimum number of meanings with the help of which you can accomplish this task, to have a set of paraphrases which jointly will be able to account for the whole set of data. Actually, the best study of corpus, I think, the most detailed, the most thorough set of study of a word which is corpus-based, maybe it's a unique study, is Cliff Goddard's study of the English word forget. He analysed hundreds, all the examples of using the word forget plus unforgettable, and so on, from COBUILD. And he came up with quite a number of frames and meanings related to these particular frames. And he showed that the explications that he came up with-we often use the word 'explication' rather than 'definition' because of the form, but ultimately, this doesn't matter - the set of explications that he proposes accounts for all the examples of the sentences with the word forget in this large English database (Goddard 2007). ${ }^{1}$ So that's the way to go, we believe. And I tried to do the same with evidence, and the explications can be complex and quite long, and then you have the practical question of how much of it should be included in a dictionary. This depends on the type of dictionary. If you want a pocket dictionary which people could use in a hurry, then obviously, you have to abbreviate. But you can't really abbreviate, it seems to me, effectively, if you don't know what it is that you are abbreviating. So for example, in the case of evidence, you have to have a set of full meanings, well explicated meanings in order to abbreviate for practical purposes. So, I think, I came up with something like five meanings, although I studied evidence in a historical perspective, so not all of them are used actively, although native speakers who read the literature, may also recognize some of the older ones. And just more recently, I've done also a very large study of another English cultural keyword, experience. Again, you can't really even come up with a hypothesis about the meaning of the word experience unless you decide how many different meanings you are going to recognize. And then, you link these different meanings with certain frames, and you test those explications against a large set of examples which you'll find in a database like the COBUILD corpus. That's the way we work now (Wierzbicka 2002a).

Andor: How do you think words representing conceptually purely frame-based lexis can or should be defined in dictionaries? I am thinking of words such as ufo, chimera, unicorn, mermaid, and others, even including marmot, and in many cultures even tiger, concerning which ordinary native speakers typically lack conceptually based, scenic or script-based knowledge, mainly due to lack of direct experience. In such cases reliance on some sort of folk categorization, everyday conceptualization, and on the emerging tacit knowledge would certainly be absolutely misleading.

Wierzbicka: I'm not sure I understand. First of all, is there any essential difference, from the point of view of meaning, between a unicorn and a tiger? That's actually an interesting question. You mean...

Andor: What I mean to ask, for instance, about tigers, is that people living in Bengal may have an absolutely different sort of knowledge due to their direct experience than, for instance, we, Hungarians have, who can only see tigers in films, can read about them, maybe we can see tigers in the zoo. So what I'm saying is a sort of issue, according to which our encyclopaedically-based knowledge is absolutely different from

${ }^{1}$ At the time of recording the interview with Anna Wierzbicka, Cliff Goddard's study on the verb forget was only available in manuscript form. It was subsequently published as Goddard (2007). 
that of people in Bengal, India and so on, who may have a different sort of attitude to and experience with tigers.

Wierzbicka: I see.

Andor: And this is also the case with the notion and word marmot. Many people have never seen a marmot, they do not even know what marmots are. But they may have read about marmots, they may even be using phrases including the word marmot, in saying, for instance $X$ sleeps like a marmot, and so on. So these people have a different sort of conceptual background, consequently, they have a different sort of depth of knowledge about the word than people who have been exposed to direct experience, who, I would say, have 'scenic knowledge' about marmots and not 'framebased knowledge', which, I would say, is a represented type of knowledge gained via reading and so on. So this is exactly why I brought up ufos, unicorns, and so on, for the case of people who definitely don't have or cannot have any form of scenic-based knowledge, because of lack of exposure, lack of direct experience. How can this folkbased knowledge be matched with dictionary representation? This is, actually, a sort of very interesting topic that I have been working on. I've been analyzing characteristic features of animals as they are represented in jokes, for instance (Andor 2002).

Wierzbicka: Right. Well, we are talking about words, not about tigers. As semanticists, we are not interested in tigers at all. We are interested in the English word tiger. And, of course, the Hungarian word which is the closest counterpart of tiger may have a different meaning from the English word tiger. So I would not compare here Hungarians with speakers of English, or Polish, and so on. So, let's just talk about English words at the moment. Actually, this is something which, I think, was discussed in Lexicography and Conceptual Analysis a long time ago. Comparing, for example, English with Russian, if you take the words like donkey and ass - and I never worked out exactly what the difference is. In Russian, they have osël and they have $i s ̌ a k$, which correspond to what biologists might tell you is the same kind of animal. But the cultural knowledge encoded in these two words is different, because there are certain ways of thinking about the animals of this kind which are linked with these particular words. So as semanticists, we have to analyse the knowledge, the assumptions, and the ideas and the prejudices, what have you, associated with one particular word such as $i \check{s} a k$, which is one particular kind of animal, and this animal works very hard without complaining, carries burdens and so on. Whereas osël, as I say, roughly, in a dictionary you would find that they both mean donkey.

Andor: This is in fact why I asked you this question. That is, how can a dictionary overcome difficulties of this kind?

Wierzbicka: The dictionary just has to articulate the whole knowledge which is linked with a particular word. So a dictionary definition of $i s ̌ a k$ would overlap, but would not be identical with a dictionary definition of osël. Osël is a stupid animal, for example, whereas $i \check{s} a k$ is not seen as stupid but is seen as hard-working. So since we are just articulating all the ideas, it's not a question of knowledge, because it's also ideas, and perhaps prejudices that speakers of a particular language link with a particular word. We just have to list all these ideas which are linked with a particular word. So, for example, kangaroo. What's a kangaroo? I don't know. Well, Australians certainly have more experience with kangaroos than speakers of English in Europe. But I think the word probably means, I imagine that the word means the same in Australian English and in British English, that a kangaroo is a particular kind of animal. And then you 
say something about the pouch when using this word, that a female kangaroo, or a mother kangaroo can carry a baby kangaroo in her pouch. Well, this is what you tell to a child. If a child asked you about what a kangaroo is, what would you tell them? You would say such kind of animal, you would say that mothers have a pouch, you would say that they can jump in a funny way because maybe their hind legs are longer than their front legs, which look like paws, and you say a few things like these, and that is what the meaning of the word kangaroo is. And the fact that zoologists may have more knowledge about them than non-zoologists or somebody who knows them, who has come across many kangaroos in their lives, it's not necessarily relevant here.

Andor: It's technical knowledge. It's expert knowledge.

Wierzbicka: It's technical knowledge, yes. But it's not just a question of technical knowledge. When you use the word kangaroo, you rely on what you think other people link with this word, when they use this word to communicate. So you can only put, so to speak, into what you are conveying with the word kangaroo, what you imagine other speakers of the language would also link with this particular word. Well, this is necessarily different in one respect from explicating the meaning of a word like unicorn. Because in the case of unicorn, well, maybe you wouldn't say it's a kind of animal, but I think it is also part of the general knowledge linked with the word unicorn that actually, they don't exist. That there are no such animals. But people have thought that there is a kind of animals which have only one horn. You would put this into the definition.

Andor: But the great specificity of this mythological kind of animal with language users is that they seem to associate and attribute to it the kinds of features and typical actions that they would associate with horses. So there is a kind of overlapping - I did experimentation on this (Andor 2001, 22-3) - , like what sort of 'jumping' would unicorns, horses and ponies do. The results show that people seem to overgeneralize aspects and knowledge about attributes and typical actions of horses over to unicorns, just for this similarity. And for some reason, interestingly, they forget about the key point of their single horn during this sort of association. So to me the strange thing is that they do not even roughly know the potential kinds of actions that unicorns would do, because of lack of proper reading, maybe. However, they overgeneralize their everyday, common, folk knowledge, as you say, about horses, to the kinds of actions that unicorns, in their view, would or could potentially perform. This is what I call frame-based knowledge. They overgeneralize because of their sets of frames in their minds. In lack of direct experience about unicorns, they do have, usually, a lot of direct experience, scenic knowledge, as I call it, about horses.

Wierzbicka: Yes. But is it essentially different from zebra? For a child who has never seen a zebra, but they know the word zebra, because they have looked at pictures and they read in children's books, they know that a zebra is a kind of funny animal which looks like a horse but has stripes. That's part of the meaning of zebra, and you don't really need to have seen zebras at all to have this knowledge, and if somebody doesn't know that the zebra is a kind of animal that looks like a horse but is not a horse because it has stripes, then I would say, then you don't know what the word zebra means. That's part of the meaning of the word zebra, which is really unrelated to whether you've seen zebras in fact or not.

Andor: How about their behavior and their actions? 
Wierzbicka: Well, you have to look at the individual words. Because different words are linked; if we are talking about different kinds of animals, then I think, usually the name of a kind of animal is linked with certain assumptions about their behavior. But the number of assumptions differs hugely depending on the cultural familiarity of a particular animal. So, if we want to define $d o g$ and cat-and these are words which I did work on in Lexicography and Conceptual Analysis - there is a huge number of assumptions about how they behave, which everybody would know who knows the meaning of the words cat and dog. In the case of kangaroos, what would all native speakers of English know who have the word kangaroo in their active vocabulary? They would know about the pouch, and perhaps they would know about the funny kind of jumping. And what would they know about zebras other than that they look like horses? They would also know about the stripes.

Andor: Tigers also have stripes.

Wierzbicka: But in the case of tigers you would say that these are big kind of animals which look like cats. Actually, I think that the word cat is part of the meaning of the word tiger, not because tigers are big cats. They are not big cats in non-technical language. They are not cats. But they are big animals that look like cats in certain respects. But we do know quite a lot about the behavior of tigers as speakers of English. But in the case of zebras, I don't think really we do know a lot about them. We know that maybe you can see them in zoos, they are sort of exotic animals, maybe they live in Africa, and they have stripes. And not much about their behavior at all. So it certainly depends on the word.

Andor: Identifying semantic primitives has constituted perhaps the most important topic of your research during the past few decades, and the NMS model is certainly based on them. Concerning the linguistic status and character of the primes and the criteria of their identification, you, and also Goddard state the following: (i) they are indivisible and indefinable (ii) they have a high frequency of occurrence in the given language, (iii) they represent universal concepts and have a universal status linguistically (i.e., they function as basic constituents of the universal lexicon), (iv) they tend to be represented and thus to occur in every single language, (v) their meanings are essential for explicating the meanings of other expressions and grammatical constructions, (vi) they can be used easily in reductive paraphrasing - the method of interpreting and identifying the meaning of linguistic expressions of varying length (Goddard 2002b, 6, 16-7; Wierzbicka 1996, 28-111, 121-2). The identification of primes requires a lot of experimental testing. My first question here concerns the details of testing, the process of justification and verification as a result of which a lexical element is identified as a prime and is added to the list. In the beginning phase of your research you had only about a third of the present number of primes. Now there are about 60 in NSM theory. How, and on what grounds is a piece of the lexis selected to be tested for a prime status? For instance, DIE was added to the list fairly recently. Why so late? Furthermore, DIE has a large number of synonyms in various languages. It can be justified by testing that of the set (or lexical field, as some people may still call it), that DIE serves as a prototype due to the nature of the cluster of its features among 'verbs of dying', as it satisfies relative interchangeability, neutrality of content, it is the least frame-dependent member of the set, among others. However, DIE, of course, also has its very unique features in the set, very actively functioning when this, rather than another verb from the set (for instance, pass away) is selected for a piece of discourse in a given register, just to name a few of the vital conditions of its use. Let me ask, 
as an addition to my primary question concerning the criteria of identification and selection, what were the specific conditions and factors of identifying DIE as a prime at a given stage of NSM research?

Wierzbicka: Thank you for asking this question. Well, you mentioned the number of criteria and I just notice that one of these criteria I would not really want to, I would not really regard it as such, and this is the criterion of frequency. As it happens empirically, semantic primes established on other grounds, still we'll find that they usually are very frequent in languages. But this is not a criterion for word choice. So the basic criterion is that we'll try again. This is Leibniz's methodology: trial and error. We start to explicate all the words, all meanings, and our assumption is again Occam's Razor. We try to find the minimum number of concepts in terms of which you could deal with everything. That's why we started with so few. Well, I started with 13 or 14, you know, in 1972 (Wierzbicka 1972), and now we actually really think that now we probably have reached the limit, which is about $65-$ you mentioned $60-\mathrm{I}$ think now we are close to 65 , and we don't really expect any more, at this stage.

Andor: I am really very interested to know why you don't expect any more.

Wierzbicka: I can tell you why. I am not saying that it's absolutely impossible that one or two more will be identified. But whatever the set is, we constantly test it by analyzing different semantic domains. Can we deal with this domain? Can we deal with that domain, in terms of the set we have? So, for example, in my book What Did Jesus Mean (Wierzbicka 2001) I analyzed a large number of ideas, not just words, but ideas essentially, the meaning of the Gospels, Christianity, and so on. And one of my questions was, and the same question: Is the set that we have sufficient for explicating what I have come to see as the meaning of Jesus's teaching? And I found that, well, it was probably almost sufficient, but there are still certain elements or certain frames which were missing, and which were necessary, such as DOING SOMETHING GOOD FOR SOMEONE. So these were introduced, because tentatively, we seem to need these elements, or these combinations in order to deal with this particular domain. But before we accept them, we test them for other languages. We say, OK, in English you seem to need these elements to deal with religion, or you seem to need these elements to deal with - recently we have done a lot of work on - verbs of physical activity, like cutting, or chopping, and digging, and so on. And you do hundreds of explications just to test whether your lexicon of primes is sufficient. And you may come to the conclusion that something seems to be missing, so we add something which would help us to solve this particular problem. And then you have to test it against a large number of other languages, and if it works, well, then we may tentatively accept that this is also a universal element. If it's not, we go back to the drawing board and we say, well, we have to solve our problem in some other way.

You asked about DIE. DIE wasn't there because we had a very small set, and at the beginning anything which seemed to be decomposable was not put on the list. But we had to do a lot more work and it has just become clear that there is no good way of analyzing DIE. So, for example, there are, of course, various alternative expressions, especially euphemisms, or words, which express a certain respect for people. But if you want to say Old people die, a fairly important human meaning, which I would expect you can express in any language, again, we have tested it, but there are other languages. It seems plausible to say that people in our societies are interested in the idea that old people die. So, how could you say it? How could you express this meaning, if you didn't have a word for DIE? 
Andor: How about perish, for instance?

Wierzbicka: Old people perish.

Andor: But perish has, kind of, different features. You tend to use perish in a given context, rather than using die.

Wierzbicka: Look! Either perish means the same as die, in which case it is just an allolex — what we call 'allolex', just a variant —or it has some other aspects of meaning, and then you need to explicate it. I think the range of use of perish is much narrower than that of die, so for example, if you say, My grandmother who was 100 years old died last year, OK, would you say, My grandmother who was 100 perished last year? You wouldn't say it.

Andor: OK, but you would say, Over a thousand people perished in the disaster. And you would also say, Thousands of people died in the disaster. And that's exactly what I'm saying, that we pick the verb die because it is frame-neutral, therefore, it is interchangeable, but not vice versa. But in a lot of cases you would not tend to use it as a substitute for pass away, because pass away is salient with certain kinds of registers and certain types of texts. And in quite a lot of cases, and I tested that as well, as a matter of fact (Andor 2005b), it might refer to a much more peaceful, expectable case of death than the kind of death that is, for instance, characterized by die.

Wierzbicka: Exactly. So that's why I think that a word like pass away or perish has a more complex meaning than die. Die is just one part of the meaning of perish and one part of the meaning of pass away. You would not say that The newborn baby passed away. You would just say, The baby died, or you could sometimes say: Unfortunately, tragically, a baby can die in the mother's womb. You wouldn't say: A baby passed away in the mother's womb. So we cannot really replace die with perish or pass away, but we can explicate pass away using the word die, plus adding certain components. You have mentioned some of them, which are probably right.

Andor: Because die is the one that carries a prototypical meaning. It serves as a prototype.

Wierzbicka: Well, that's one way of putting it. I don't even know whether we have to refer here to a prototype. It has a simpler meaning. It's a meaning which you cannot explicate, whereas you can explicate pass away by using die plus a few other semantic components taken from our list of universal concepts. So we just have no way of paraphrasing Old people die in terms of simpler concepts. We just acknowledge the fact that we cannot do it, and moreover, as you look at other languages, so far in every single language that we looked at, there is a way of saying Old people die, which doesn't carry any additional assumptions or implications different from those embedded in the English expression. So we just recognize the fact, first of all, that you cannot explicate it in simple terms, and second, that you find it in all languages. And this then applies to the rival sort of new elements which have appeared on the list.

Andor: I guess that beyond the factors listed in my previous question concerning techniques of identifying the primitives, a given depth of abstraction is also needed in their identification. How can the required level of abstraction be identified? I think this is a critical, highly relevant issue to be discussed. Do we apply the same principles of abstraction to decide on the prime status of a lexical relation or item? The case of GOOD offers good grounds for discussing this issue. GOOD is a member of your list of primitives, and its meaning is practically impossible to define, it is indivisible, indefinable. Unless we take a pragmatic stance and investigate via paraphrasing how it 
can be interpreted in various contexts with their key words, interpreting the meaning of GOOD in structures such as good knife, good friend, good book, good voice, good tea, etc. Paraphrasing can reveal differing content in each such case. This is a highly pragmatic issue, I believe, which calls for the examination of inferential pragmatic processing, a field that, as Goddard says, calls for further investigation (Goddard 2002a, 315). Isn't it the case that GOOD as a concept has a certain set of lexical expressions, including the lexical item good (along the lines of Wierzbicka 1999, 35), and others such as correct, appropriate, pleasant, then also lexical items that are in a given type of synonymous relation with good such as outstanding, excellent, superb, which items are all gradationally related to good, or even items such as sharp, tasty, interesting, which expressions show different types and grades of frame dependence, but which serve well in paraphrasing GOODness? With a certain level of abstraction and testing of prototypicality it can be justified that among these good is the one that is most neutral, the least frame dependent, reveals the lowest number of lexical gaps in collocability with nominals, hence it is the one that has the highest rate of prototypicality, as a result of which it is the best candidate for the semantic prime status, coping with the other factors, criteria mentioned above. Similar issues arise related to the prime status of the concept VERY and the intensifiers very, awfully, terribly, horribly, terrifically and other members of the same group, revealing a varied case of occurring lexical gaps in their adjectival collocations, and also a varied picture of expressing negative vs. positive polarity, with an interesting variation of shifts in the latter domain as pointed out by Andor (2003), Partington (1993) and others.

Wierzbicka: Again, an interesting and complex question. Let us just discuss the case of GOOD, first of all. I think it's very important. We have certain canonical contexts, canonical sentences, meanings which we can express in all languages. And I don't just refer here to the meaning of words, but to certain ideas, such as I DON'T WANT BAD THINGS TO HAPPEN TO ME, which seems to be a universal human idea. People are concerned in all societies with this, especially with I DON'T WANT BAD THINGS TO HAPPEN TO OTHER PEOPLE, for instance, women don't want bad things to happen to their children. The idea can be translated from any language into any language, SOMETHING BAD CAN HAPPEN TO THESE PEOPLE, I DON'T WANT BAD THINGS TO HAPPEN TO THESE PEOPLE. Similarly with good, I WANT GOOD THINGS TO HAPPEN TO THESE PEOPLE, I WANT GOOD THINGS TO HAPPEN TO ME, I WANT GOOD THINGS TO HAPPEN TO SOME OTHER PEOPLE, such as my children. Well, this is good. And of the words which you mentioned as possible quasi synonyms...

Andor: Or interpretations.

Wierzbicka: Most of them would not be applicable in this context. So I don't think we should start with a good knife or good food. Let's just start with precisely these key contexts of GOOD THINGS CAN HAPPEN TO ALL PEOPLE. BAD THINGS CAN HAPPEN TO ALL PEOPLE. These are tremendously important human ideas. And in all languages you can say them. In all languages you know what word to use, how to translate it. It's not a question that you want outstanding things to happen or that you want excellent things to happen, and that you want superior things to happen, or effective things, you want GOOD things to happen to your children. And you don't want BAD things to happen to your children. So you can choose those sentences and identify your basic exponents of GOOD and BAD on the basis of these contexts. Also the idea of IT IS GOOD IF SOMEONE WANTS TO DO GOOD THINGS FOR 
OTHER PEOPLE, a crucial ethical idea. Translate it into other languages and you will find your basic exponent for GOOD. And usually there are no synonyms to be used in this sort of key context. And then you can notice that in some other contexts, such as when we talked about knives, for example, you can say a good knife or a sharp knife, but all those other words, which in practice can be used for praising various kinds of objects, usually you have words which can be explicated, which are quite complex, including words like outstanding and excellent, it's not just GOOD, there's more to it, and we can explicate them. So I didn't want even to talk here about prototypes or about frames. I would just talk about elementary meanings which cannot be explicated, and more complex meanings which can be explicated. Probably the same would apply to all those quasi synonyms for VERY.

Andor: My next question concerns the syntactic status of primitives. NSM claims that there exists a core syntax in language that is universal and serves as an underlying source for the technique of interpretation via reductive paraphrasing. You have used the technique successfully in identifying/interpreting lexical meaning, and even for the purposes of interpreting discourse and texts. What strikes me, however, is the syntactic structure, which is claimed to grasp universal relations, but in reality it contains syntactic devices that are highly language specific: structuring according to conventional roles, including infinitives (see, for instance your analysis of love your enemies on pp. 7-8. of your book What Did Jesus Mean? (Wierzbicka 2001)). The interpretation given in paraphrases there and elsewhere in the book contains prepositional phrases of the type that were not included in your list of primitives (do good/bad things to this person vs. for this person, for instance). On the other hand, for instance, Goddard warns us $(2002 \mathrm{~b}, 8-9)$ that imperatives are not to be used in reductive paraphrasing because they are not universal. Another difficulty I find in the paraphrasing technique is again the pragmatic depth of abstraction. For instance, in the same text sample enemy is paraphrased as someone/the person [who] wants to do bad things to you. Is this a correct paraphrase for 'enemy'?

Could you clarify to me more precisely how the operation of NSM syntax (grammar) should be interpreted?

Wierzbicka: We test the adequacy of NSM syntax by doing explications in many different languages. So for example, just recently, I think, I mentioned that we did work on verbs of physical activity, such as Somebody was cutting something, Somebody was chopping something, or slicing something, and so on, and we arrived at quite complex and long explications, and then we tried to translate the explications, so to speak, word for word into a number of other languages. And in the process of doing this we discovered that some English explications which seemed very very plausible and natural in English didn't work in all respects equally well in Polish or in Japanese. Even in my native Polish, but also in Japanese. So for us this was a signal that something was wrong with the English explications. And, of course, there are many expressions, frames, or constructions in English which sound deceptively simple and natural, but which are not really translatable into other languages. Such as, for example, what will you do about it. Well, try to say do about it in other languages and you will find that you can't. Or, how do you feel about it? Again, it sounds simple, but it's English specific. So this is how we find out that there is something which is language specific in our explications, by doing equivalent explications in a large number of languages. We will never come across something which doesn't work in any other language that we can work on. We go back to the original explication and revise it accordingly. For 
example, recently we found that a number of our English explications which seemed to us very very good, well, when we tried to translate them into other languages, they didn't work so well, because, as we discovered, we were relying on English articles, saying something about the place where someone was. Especially, when we were doing a lot of work on verbs like walking, running and jumping, and so on, and we had long explications which we found quite successful, but when we tried to translate them into another language which doesn't have articles you realize that suddenly there is a difference between the place where someone was and a place where someone was, and you have to redo it, because the syntax doesn't match. We are very much concerned about universality of syntax. So we do care about this and we revise our explications in such a way as to make them fully translatable.

So, going back to the example that you quoted, SOMEONE WANTS TO DO SOMETHING GOOD FOR SOMEONE ELSE. We think this synthesis is a very important frame. DO SOMETHING GOOD FOR ANOTHER PERSON, or IT IS GOOD IF SOMEONE WANTS TO DO GOOD THINGS FOR OTHER PEOPLE. I think that from an ethical and religious point of view, this is a very important idea. So to me, personally, it was extremely important to see whether this particular idea can be translated into other languages. In other words, can we say in other languages that SOMEONE WANTS TO DO GOOD FOR OTHER PEOPLE? And yes, in our experience this works very well. Whether it is a prepositional phrase, the mechanics of it don't matter. Maybe in another language you'll find a suffix for the idea that YOU ARE DOING SOMETHING FOR SOMEONE ELSE. It doesn't have to be a preposition. That doesn't matter. This is a good universal frame in our experience so far, tested across quite a number of languages. Similarly, THIS PERSON DID SOMETHING BAD TO THAT OTHER PERSON. Again, it's an important question whether this idea can be translated into other languages, and whether you find some syntactic means for saying precisely this. Not just that 'you did something bad', but that 'you did something bad to another person'. So far we have not had any problems. You find it in languages. The syntax here is perfectly translatable, and so far we would claim that these expressions, these configurations, these various syntactic valencies are universal.

And then you mentioned enemy and asked if this was a good definition. Well, it's not a very good definition, it's an approximation. The formula that you quoted for enemy: SOMEONE OR THE PERSON WHO WANTS TO DO BAD THINGS TO YOU. Well, that's certainly insufficient. Also syntactically, someone who is not a universal syntactic frame. So I wouldn't use someone who. But the idea that this person, your enemy, IF SOMEONE IS YOUR ENEMY, THIS PERSON WANTS TO DO BAD THINGS TO YOU, I don't know. It seems to me plausible, but I'm not sure whether it is part of the meaning of enemy. Do you think that this is not part of the meaning of enemy?

Andor: When I quoted it, of course, I did not have who, I just added who in brackets for the interpretation.

Wierzbicka: I see. So there's a syntactic problem. This was not proposed as an accurate explication, it was just a throw away approximation. Often we do need approximations. But it's not a satisfactory idea. We are talking about the English word enemy, because you also have opponent, for example. It's important to explicate them in such a way, to identify similarities and differences between opponents and enemies, and there could be other related words as well. I think, it seems to me that 
the word enemy does imply, among other things, that THIS PERSON WANTS TO DO BAD THINGS TO YOU. Maybe there's also an idea that THIS PERSON WANTS BAD THINGS TO HAPPEN TO YOU, I'm not sure.

Andor: Whereas opponent may not imply all this.

Wierzbicka: That's right. So it's not sufficient. But I think we are on the right track. That's what I would say.

Andor: OK. I would also like to ask you about your thinking about the status of key words. You devoted a whole book to studying key words (Wierzbicka 1997) embedded in culture. You seem to favor a socio-cultural approach to identifying key words, at best supported by studying their frequency of occurrence in discourse. Your definition sounds like this:

“'Key words' are words which are particularly important and revealing in a given culture. [...] There is no finite set of such words in a language, and there is no 'objective discovery procedure' for identifying them. To show that a particular word is of special importance in a given culture, one has to make a case for it."

(Wierzbicka 1997, 15-6)

Corpus linguists such as Mike Scott also consider frequency of occurrence to be decisive on key word status (Scott 2002; 2004; Scott-Tribble 2006). The approach you offer is better, as it considers frequency within given semantic domains (Wierzbicka 1997, 16). But in order to be able to test key word status, how can we delineate the borderlines of types of semantic domains, which in itself is a rather vague term to be used. The other issue that arises here concerns factors influencing the choice and role of key words: age, time period, and register differences are certainly among them. I think that all these have a great influence upon the choice of key words as understood in terms of your thinking. How can this be matched with the role played by cultural universals and specificities?

In text linguistics and discourse analysis, key words have a different status. They are markers, critical building blocks of coherence, the highest set of norms over textuality. It is key words that lexically organize discourse via their frame- and script instigating and frame- and script mapping capacity (Andor 1989, 31-5). Which role is critically different from the one you attribute to them. Do you think that the two approaches can show any correspondence or can be merged? Perhaps the notion of scripts can bridge differences in interpreting their status.

Wierzbicka: Well, I think, in principle, we need to explicate all the words that a language has. So there is no particular, strictly delimited category of keywords. It seems to me that one way to approach this question is a practical way. For example, you have immigrants to English speaking countries. Actually, this is how I think about it a lot, you know. You have so many millions of immigrants coming to live in English speaking countries. They have to learn the English vocabulary, well, not all of it at the same time. They have to acquire new concepts linked with these new words. Which words are particularly important to teach to immigrants? So, from a practical point of view, I think this is a good way of approaching the question. These words don't necessarily have to be nouns, or words recognized as names of values, because in a sense, it seems to me, it's also extremely important to learn certain discourse markers. Whether you would want to call them discourse markers, keywords, or not, well, I don't care, but you know, if you want to speak English, if you want to live in an 
English speaking country, a word like $O K$ is textually important. Now, do you want to call it a keyword? I don't know, it does not really matter what we call it. We have to recognize $O K$, that it is a very very important English word, and you have to teach immigrants how to use it and what it means. And actually, that's probably one of the words that immigrants being in English speaking countries pick up first, because people are aware of how important it is. It's much less obvious that a word like evidence is an important English cultural word, but if you study it in detail, you see that it is, and you just see that it doesn't have equivalents in other languages, and then, if you study it more closely, you discover how important it is and how exactly it developed (Wierzbicka in press).

Another word which I mentioned earlier, and I've done quite a lot of work on recently, is experience. Again, it's not obvious that these words are very important in English speaking countries, but if you look at them more closely, you discover that these are very important tools for people to think with and to argue with. So you really have to discover what these words are. For example, if you are a Hungarian or a Polish linguist and you publish in English, you learn that it's very important to use in your writing and in your thinking the concept of evidence; there is no word for evidence in Polish, and I bet that there's no word for evidence in Hungarian. We have words like proofs, but we don't have a word like evidence. But you learn to think and to argue in terms of the concept evidence. It's an English cultural keyword, I would say.

Andor: So your notion of 'keyword' has a different status. You are talking about 'cultural keywords', and the sort of understanding that I want to suggest is a sort of textual organizer, which is a topical organizer of texts. Now, that's a different kind of term, but the function is also that of a 'keyword'. Now, how your kind of notion can be merged with this other kind of concept, understanding about keywords, I think, would definitely require a lot of empirical work. How, for instance, $O K$ occurs in different types of discourse and what's the role, if it has any textual organizing role at all, is very difficult to ascertain.

Wierzbicka: But you know, these things are related. Like, for example, in a book, my latest book, which is due out very shortly, I have a whole chapter (Wierzbicka 2006, Chapter 3, 61-102), ${ }^{2}$ and I also published an article as a preliminary version to the book in Discourse Studies (Wierzbicka 2002b), and this is on right and wrong. I would say that these are English cultural keywords. These are terribly important concepts: when people write about ethics in English, lots of people, philosophers, when they write about ethics in English, they say that the distinction between right and wrong is a fundamental human distinction. This is what defines us as humans, as distinct from animals. And as I pointed out in this article and the book, in other languages, for example in Polish, there is no such distinction. All languages have a distinction between good and bad. But the notions of right and wrong, as distinct from good and bad, are really artefacts, cognitive artefacts of Anglo-culture. So I think, we could certainly say that right and wrong are English cultural keywords. But also, especially the word right has come to play an extremely important role as a discourse marker. It is almost as important as $O K$, you know. It's difficult to conduct a conversation in English without saying Right, Right, Right. It's an extremely important discourse marker. And I think, as I tried to show in the article and in the book chapter,

2 The book, entitled English: Meaning and culture, was published just one month after the recording of the present interview. 
there is a semantic link between the use of right as a discourse marker and the use of right as a basic ethical concept. So, again, we have a sort of practical considerations here. We can use terminology in the way which seems most practical, but essentially, I'm talking about words, which for one reason or another are particularly important in a given society, and which are unique to this society. We have to somehow focus on those and teach immigrants, for example, how these words operate. Which of them will be called 'keywords' and which will be called important discourse markers, in essence, is not such an important question. The important question is that we identify those words which are culture-specific, and which are, for one reason or another, particularly important for people to function effectively in a particular society.

Andor: You and your coworkers have devoted special attention to analyzing what you call cultural scripts. However, your understanding of the notion of scripts is significantly different, I believe, from that found in the cognitivist, AI, or frame semanticist literature. Scripts in their sense constitute a type of basically vertically structured conceptual structure representing stereotyped situations in a sequential order of typical actions. Of course, such sequences are greatly dependent on cultural stereotypes. In your understanding, too, scripts are culturally dependent, and key words have a critical role in their content, they constitute the lexical core of a "whole network of culture specific cultural scripts" (Wierzbicka 1997, 17). Your approach to scripts concentrates on the socio-cognitive, cultural, lexical aspects, on the lexical representation of cultural values and norms, culture-specific speech practices and interactional norms, not so much on the sequential properties of the typology of actions (Goddard-Wierzbicka 2004, 153-4). Can the two approaches be merged? I believe, they can. One can have a sequential understanding in terms of behavioral routines of 'scripts of sincerity', 'scripts of warmth', 'scripts of spontaneity' - your examples are taken from p. 241 of Emotions Across Languages and Cultures (Wierzbicka 1999).

Wierzbicka: Again, I would not want to argue about terminology, as I, personally, am quite attached to the word 'script', but some other people use it in another sense, and they can feel free to do so. So this is not really a difference of opinion. That's a difference of terminology. We use the term 'script', and in particular, 'cultural script' for explicating assumptions shared by many people in a given society, mainly about norms, that is to say, IT IS GOOD TO DO such and such, IT IS BAD TO DO such and such, IT IS GOOD TO SAY such and such, IT IS BAD TO SAY such and such, or sometimes I CAN DO such and such, sometimes I CAN'T DO such and such, GOOD or BAD. We think that it's extremely important for practical reasons for intercultural communication and cross-cultural understanding to be able to identify and correctly articulate those norms which we want to call 'cultural scripts'.

Now, this other use of the term 'script', for instance, along the lines of Schank and Abelson (1977), that's certainly a different sense. They don't really start from the meaning of words or meaning of expressions. But there are certain words and expressions which do embody what I would prefer to call certain scenarios, which refer to a sequence of components. We don't call them scripts, because they are different from cultural norms for which we use the term 'cultural scripts'. That's all. For example, you mentioned my book on emotions. To explicate accurately the meaning of certain emotion terms, such as Schadenfreude (Wierzbicka 1999, 103-4), something that I worked on, there is a certain set of items what you might call 'scenario', revenge, or envy, for instance, you know. Sequences can be very very important for the meaning of certain words or certain expressions. So we try to identify them, we try to articulate 
them and keep them in the sequence which the word suggests. This is extremely different from what people like Schank and Abelson do, because as I mentioned, they don't start from linguistic expressions. So they say, there's a certain routine, and when people do this, they operate in the following way. Well, that's fine, I have absolutely no problem with it. But it's really a sort of different task from identifying the meaning of words, even of words like, say, revenge, it's different from articulating a culture of norms, which we call 'cultural scripts'.

Andor: A couple of years ago, in 2000, I had a chance to listen to your lecture presented at a workshop organized on the typology of word classes, where radically new approaches were introduced on the status and role of conventional types of parts of speech. Referring to work by Dixon as well as to your own work on semantic primes, you devoted special attention to discussing prototypical characteristic features of word classes, arguing for prototypical nouniness, verbness, etc. (Wierzbicka 2000). As I have not read more from you about this morphological issue since then, let me now ask you how you would relate such work to your current thinking about the semantic primes as lexis and the sort of basic, NSM grammar you are advocating.

Wierzbicka: Now, I haven't really worked any longer on this particular issue. I think what interests me here mainly is identifying semantic differences between types of words which many people regard as unimportant and superficial. I think that very often if you have a choice, for example, between what people call and adjective or a noun, and they say it doesn't matter, they mean the same thing, well, often there is a subtle semantic difference. And that's one of the things I tried to do in the paper that you referred to: trying to identify what it is, and this may be linked with a current trend in thinking. People reject what they call 'essentialism'. Like, for example, words like cripple. You wouldn't call anybody a cripple today, because people are extremely sensitive that this is offensive, whereas a hundred years ago people didn't feel there was anything offensive about it. Now people would say a word like somebody is disabled rather than use any kind of a noun. And here the sense is, I think, that if you use a noun to describe a category of people, you are somehow 'essentializing' this group. You are treating this particular property as essential to these people. So there is a sense here that whether you use a noun or an adjective, sort of, matters. And that's the kind of thing that linguists until recently didn't really recognize. And I think the intuition is right. So a category like noun may suggest a different way of conceptualizing a certain property which can also be expressed by means of an adjective, but with different implications. And similarly with emotions. In English, for example, many emotion words are adjectives, whereas in Russian their closest counterparts would be verbs. Does it matter? Well, I've looked at it in some detail, and it seems to me that it, sort of, does matter, and that we find some certain important cultural clues there. Why is it that Russians tend to talk about emotions in terms of verbs rather than in terms of adjectives? Well, I've come to the conclusion that this may be linked with different cultural attitudes to emotions and with different cultural scripts, but concerning how you deal with your feelings. So, I think that these are very interesting and important grammatical clues to cross-linguistic and cross-cultural differences in attitudes, in the way of thinking, in the way of evaluating human behavior, and so on. So this is the point of view from which these differences between parts of speech, so-called, have interested me most.

Andor: All of us, lexical semanticists and researchers of pragmatics, who have followed your work with great attention for decades now, would be greatly interested to know 
about your plans for the future. What are the most important general and particular issues for developing and extending the NSM theoretical framework beyond extending it further to the investigation of a still wider range of languages? One domain that I feel should be included in testing the descriptive and explanatory capacity of the model would be the analysis of discourse and text. Your investigations of Jesus's discourse certainly point to that direction (Wierzbicka 2001). What's coming next?

Wierzbicka: Well, I have this book just coming out now, which I mentioned, entitled English: Meaning and culture (Wierzbicka 2006). The idea here is that English is becoming or has become largely a global language. And English, I think, has certain cultural assumptions embedded in it. There's a great deal of culture embedded in English. What does it mean to the world at large? What are the cultural assumptions that English brings with it? I think that especially in view of the practical importance of English now in the world, as a language of science, of business, of diplomacy, of traffic control, you know, it's just extremely important to understand what is embedded in English, and many people, I find, are generally not aware of it. People, sort of, treat English as a kind of neutral medium, or something that we can all use, and it doesn't matter, because we are using it with a culturally completely neutral meaning. I think this is extremely misleading, because we, sort of, swallow all these cultural assumptions which are embedded in English, and we regard them as something, sort of, basic to human nature. And when I read books about human emotions, human values, even illnesses, for example psychiatry, to what extent it depends on cultural assumptions linked with the English language. I think all this should be shown. Of course, particularly in the case of English, I think we need to understand what the cultural baggage is, which is carried to the whole world with English itself. Well, only because we want to be free, we don't want the language to use us, we want to use the language rather than be used by it, I think we have to be aware of what the language brings with it. And this is something you can only do, I think, if you analyse, explore English through universal human concepts. And nobody else is doing this. First of all, you have to have an independently established and independently tested set of universal human concepts. And then you can use it to explore culture-specific aspects of all languages, but in particular, of English, because of this huge international importance of English as a global language. So, I've published one book on it, but I have a large project which continues this work. And I am also very interested in looking at it in a historical perspective. I find it absolutely fascinating. How did it happen that this whole culture which is now invisible because it's embedded in English that we all use, where does it come from? How did it happen? What was it that led to all those cultural assumptions which are now playing such an important role in global discourse? And people talk about world-wide understanding, and this is the subtitle of our conference here, intercultural pragmatics and world-wide understanding. I think that they cannot have world-wide understanding without having a shared conceptual language, which is not dependent on any particular culture. So that's why, I think, that for worldwide understanding we do have some sort of shared human conceptual vocabulary, and I think that the set of 65 or so concepts which we have identified can serve as a rudiment of such a vocabulary. And using those concepts we can also explore more complex vocabulary, such as the vocabulary that English brings with it. Without this I don't really think we have true world-wide understanding. We have to question and explore concepts like 'experience', like 'evidence', 'rights', 'tolerance', I mean, all those concepts, including 'right or 'wrong', 'reasonable', 'fair', and so on, concepts which 
are regarded as so basic and so important that they are not questioned. They are not really general human concepts, they are Anglo-concepts. And we can understand them, explore them and teach them, if we want to, through universal human concepts. So, semantics ultimately, for me, is a question of practical usefulness, and there have been great problems which we can help solve through the use of universal human concepts.

Andor: Thank you, Anna, very much for giving me this stimulating interview. Let me tell you that I'm very much indebted, because it has increased my knowledge and understanding of your model greatly. Let me, at the same time, also express here my and quite a few of my colleagues' interest in, maybe, even contributing to your theory for future, because certainly, my native language, Hungarian, is not one of those very many languages that you mentioned in your list of studies. I'm not sure, but I think that I have not found any Finno-Ugric language on the list either. So studying Hungarian in the framework of your model would certainly contribute to justifying the typological relevance of your investigations.

Wierzbicka: I do hope, indeed, that you and your colleagues will work on Hungarian from this point of view and that you will test our work and our hypotheses through Hungarian. That will be very good. Thank you.

József Andor

Department of English Linguistics University of Pécs Hungary andor@btk.pte.hu

\section{References}

Andor, József 1989. Strategies, tactics and realistic methods of text analysis. In: Wolfgang Heydrich-Fritz Neubauer-János S. Petőfi-Emel Sözer (eds): Connexity and coherence: Analysis of text and discourse (RTT 12), 28-36. Walter de Gruyter, Berlin \& New York.

Andor, József 2001. Angol és magyar, ugrást kifejező igék lexikális szemantikai és pragmatikai vizsgálata [A lexical semantic-pragmatic analysis of verbs of jumping in English and Hungarian]. In: Tamás Gecső (ed.): Kontrasztív szemantikai kutatások [Research in contrastive semantics], 7-24. Tinta Könyvkiadó, Budapest.

Andor, József 2002. On the cohesion and coherence of animal jokes: A frame-semantic analysis of narrative structure. In: Szilvia Csábi-Judit Zerkowitz (eds): Textual secrets: The message of the medium, 90-101. School of English and American Studies. Eötvös Loránd University, Budapest.

Andor, József 2003. Functional studies in the polariry and gradation of amplifier adjectives and adverbs in English. In: József Andor-József Horváth-Marianne Nikolov (eds): Studies in English theoretical and applied linguistics, 43-59. Lingua Franca Csoport, Pécs.

Andor, József 2005a. Cognitive grammar: The state of the art and related issues: An interview with Ronald Langacker. In: Acta Linguistica Hungarica 52 :341-66. 
Andor, József 2005b. Verbs of dying in English and Hungarian: A contrastive, corpusbased study of near synonymy. Paper presented at the 4th International Conference of Contrastive Linguistics.

Goddard, Cliff 2002a. The on-going development of the NSM research program. In: Cliff Goddard - Anna Wierzbicka (eds): Meaning and universal grammar: Theory and empirical findings. Vol 2, 301-21. John Benjamins, Amsterdam \& Philadelphia.

Goddard, Cliff 2002b. The search for the shared semantic core of all languages. In: Cliff Goddard - Anna Wierzbicka (eds): Meaning and universal grammar: Theory and empirical findings. Vol 1, 5-40. John Benjamins, Amsterdam \& Philadelphia.

Goddard, Cliff 2003. Whorf meets Wierzbicka: Variation and universals in language and thinking. In: Language Sciences 25 : 393-432.

Goddard, Cliff 2007. A "lexicographic portrait" of forgetting. In: Mengistu Amberber (ed.): The language of memory in a crosslinguistic perspective, 119-37. John Benjamins, Amsterdam \& Philadelphia.

Goddard, Cliff-Anna Wierzbicka 2004. Cultural scripts: What are they and what are they good for? In: Intercultural Pragmatics $1: 153-67$.

Partington, Alan 1993. Corpus evidence of language change: The case of the intensifier. In: Mona Baker - Gill Francis - Elena Tognini-Bonelli (eds): Text and technology: In honor of John Sinclair, 177-92. John Benjamins, Amsterdam \& Philadelphia.

Schank, Roger C.-Robert P. Abelson 1977. Scripts, plans, goals, and understanding: An inquiry into human knowledge structures. Lawrence Erlbaum, Hillsdale NJ.

Scott, Mike 2002. Picturing the key words of a very large corpus and their lexical upshots - or getting at the Guardian's view of the world. In: Bernhard Kettemann-Georg Marko (eds): Teaching and learning by doing corpus analysis, 43-50. Rodopi, Amsterdam.

Scott, Mike 2004. WordSmith Tools Version 4.0. Oxford University Press, Oxford

Scott, Mike-Christopher Tribble 2006. Textual patterns: Key words and corpus analysis in language education. John Benjamins, Amsterdam \& Philadelphia.

Wierzbicka, Anna 1972. Semantic primitives. Athenäum, Frankfurt am Main.

Wierzbicka, Anna 1985. Lexicography and conceptual analysis. Karoma, Ann Arbor.

Wierzbicka, Anna 1996. Semantics: Primes and universals. Oxford University Press, Oxford.

Wierzbicka, Anna 1997. Understanding cultures through their key words: English, Russian, Polish, German, Japanese. Oxford University Press, New York.

Wierzbicka, Anna 1999. Emotions across languages and cultures: Diversity and universals. Cambridge University Press, Cambridge.

Wierzbicka, Anna 2000. Lexical prototypes as a universal basis for cross-linguistic identification of "parts of speech". In: Petra M. Vogel-Bernard Comrie (eds): Approaches to the typology of word classes, 285-317. Mouton de Gruyter, Berlin \& New York.

Wierzbicka, Anna 2001. What did Jesus mean? Explaining the Sermon on the Mount and the parables in simple and universal human concepts. Oxford University Press, New York.

Acta Linguistica Hungarica 56, 2009 
Wierzbicka, Anna 2002a. Philosophy and discourse: The rise of really and the fall of truly. In: Christine Béal (ed.): Langue, discours, culture (Cahiers de Praxématique 38), 85-112. Praxiling, Montpellier.

Wierzbicka, Anna 2002b. Right and wrong: From philosophy to everyday discourse. In: Discourse Studies 4:225-52.

Wierzbicka, Anna 2006. English: Meaning and culture. Oxford University Press, Oxford.

Wierzbicka, Anna in press. Evidence, experience, sense: The hidden cultural legacy of English. Oxford University Press, New York. 